\title{
Low-lying states in even Gd isotopes studied with five-dimensional collective Hamiltonian based on covariant density functional theory
}

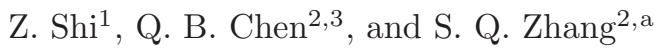 \\ 1 School of Physics and Nuclear Energy Engineering, Beihang University, Beijing 100191, China \\ 2 State Key Laboratory of Nuclear Physics and Technology, School of Physics, Peking University, Beijing 100871, China \\ 3 Physik-Department, Technische Universität München, D-85747 Garching, Germany
}

Received: date / Revised version: date

\begin{abstract}
Five-dimensional collective Hamiltonian based on the covariant density functional theory has been applied to study the the low-lying states of even-even ${ }^{148-162} \mathrm{Gd}$ isotopes. The shape evolution from ${ }^{148} \mathrm{Gd}$ to ${ }^{162} \mathrm{Gd}$ is presented. The experimental energy spectra and intraband $B(E 2)$ transition probabilities for the ${ }^{148-162} \mathrm{Gd}$ isotopes are reproduced by the present calculations. The relative $B(E 2)$ ratios in present calculations are also compared with the available interacting boson model results and experimental data. It is found that the occupations of neutron $1 i_{13 / 2}$ orbital result in the well-deformed prolate shape, and are essential for Gd isotopes.
\end{abstract}

PACS. 21.10.Re Collective levels - 21.60.Ev Collective models - 27.70.+q $150 \leq \mathrm{A} \leq 189$

\section{Introduction}

The rare-earth region, which possesses many transitional and well-deformed nuclei, is an ideal venue to study the origination of deformation and collective motion. One interesting and important example is the Gadolinium isotopes, the structures of which have attracted a large number of experimental and theoretical studies.

A well-known phenomenon in Gd isotopes is the presence of quantum phase transition. As pointed in Refs. 1, 2, ${ }^{154} \mathrm{Gd}$ locates at the $\mathrm{X}(5)$ critical point of a vibrator to axial rotor phase transition. The quantum phase transition in the $\mathrm{Gd}$ isotopes has been investigated by the potential energy surfaces (PESs) within the framework of microscopic mean-field theory. For the relativistic meanfield (RMF) calculations, the shape transition between spherical and axially deformed nuclei in rare-earth region is investigated by the microscopic quadrupole constrained RMF theory with the NL3 interaction [3, and the possible critical point nuclei are suggested to be ${ }^{150,152} \mathrm{Gd}$. For the results obtained from the relativistic Hartree-Bogoliubov (RHB) calculations with NL3 4, a relatively flat PES occurs for the ${ }^{150} \mathrm{Gd}$. Whereas, the PES of ${ }^{154} \mathrm{Gd}$ is not flat and exhibits a deeper minimum in the prolate region and a shallower minimum in the oblate region. For the non-relativistic case, a self-consistent Skyrme-HartreeFock plus BCS calculations is performed [5]. In this case, the transitional behavior for ${ }^{152,154} \mathrm{Gd}$ is found, and the

\footnotetext{
a sqzhang@pku.edu.cn
}

flat behavior, which is one of the expected characteristics of $\mathrm{X}(5)$ critical point symmetries, is not found in the PESs of the $N=90$ isotones. This is in agreement with relativistic mean-filed calculation 4]. Another non-relativistic mean-field calculations was performed with self-consistent Hartree-Fock-Bogoliubov approximation based on the finiterange and density-dependent Gogny interaction [6, including ${ }^{152,154} \mathrm{Gd}$. In the case of $\mathrm{X}(5)$ candidates, a shape transition from spherical to well-deformed nuclei was found, but the realization of the $\mathrm{X}(5)$ symmetry in terms of flat (quadratic) patterns in the $\beta_{2}(\gamma)$ potentials is uncertain [6].

Another interesting investigation in the Gd isotopes is the so-called partial dynamical symmetry (PDS) [7,8, 9 , [10. In Ref. 11, the SU(3) PDS was proposed to be used as a selection criterion for higher order terms in the general interacting boson model (IBM) Hamiltonian. Taking ${ }^{156} \mathrm{Gd}$ as an example, the SU(3) PDS improves the description of odd-even staggering of the $\gamma$ band, which is absent in the $\mathrm{SU}(3)$ dynamical symmetry. Systematic examinations of SU(3) PDS in finite nuclei have been recently carried out in Ref. 12. The relative interband $B(E 2)$ ratios of 47 deformed nuclei in the rare-earth region were first compared with the available experimental data [12, including ${ }^{158} \mathrm{Gd}$. Besides, the link between the concepts of PDS and quasi-dynamical symmetry (QDS) [13, 14 was revealed by Kremer et al. in the ground-state band of ${ }^{160} \mathrm{Gd}$ [15,16]. Such link has been further demonstrated by Van Isacker [17. 
The microscopic density functional theory (DFT), which starts from an effective nucleon-nucleon interaction and self-consistently determines the nuclear mean-field by all the independent particles inside, has achieved a lot of successes in describing both the nuclear ground state and excited state properties [18, 19,20,21,22. Based on the DFT or its covariant version CDFT, many properties of collective structures of nuclei are well described. In recent years, five-dimensional collective Hamiltonian based on the covariant density functional theory (5DCH-CDFT) 23,24 has been developed and extensively applied to describe the collective properties such as the phase transitions [25,26, [27, 28, 29, shape evolutions $330,31,32,33,34,35$ as well as the low-lying spectra along with the isotopic and isotonic chains in different mass regions $223,36,37,38,39$. The even ${ }^{152-160} \mathrm{Gd}$ isotopes have been investigated by the 5DCH-CDFT with PC-F1 parameter [23].

In the present paper, we will reinvestigate the ${ }^{148-162} \mathrm{Gd}$ isotopes in the framework of 5DCH-CDFT with the density functional PC-PK1 [39,40]. The purpose of this work is twofold: (i) By starting from $N=84$, the low-lying states for the Gd isotopes will be discussed from the 5DCHCDFT perspective, and a complete structure evolution from near spherical to well deformed nuclei will be clearly illustrated. (ii) The relative $B(E 2)$ ratio, which is an observable closely related to the PDS, will be investigated.

The paper is organized as follows. In Sec. 2 a brief introduction to the framework of $5 \mathrm{DCH}$ is given. In Sec. 3 the potential energy surfaces, excitation spectra, intraband $B(E 2)$ transition probabilities and relative $B(E 2)$ ratios etc., for even-even ${ }^{148-162} \mathrm{Gd}$ isotopes obtained from the $5 \mathrm{DCH}-\mathrm{CDFT}$ are presented. Finally, a summary is

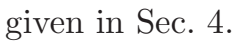

\section{Theoretical Framework}

A detailed formalism of the 5DCH has been presented in numerous articles in literatures, see, e.g. Refs. [41,23. In this subsection, for completeness, a brief introduction is presented.

The five-dimensional collective Hamiltonian, which could simultaneously treat the quadrupole vibrational and rotational excitations, is expressed in terms of the two deformation parameters $\beta$ and $\gamma$, and three Euler angles $(\phi, \theta, \psi) \equiv \Omega$ that define the orientation of the intrinsic principal axes in the laboratory frame,

$$
\hat{H}_{\text {coll }}(\beta, \gamma)=\hat{T}_{\text {vib }}(\beta, \gamma)+\hat{T}_{\text {rot }}(\beta, \gamma, \Omega)+V_{\text {coll }}(\beta, \gamma) .
$$

The three terms in $\hat{H}_{\text {coll }}(\beta, \gamma)$ are respectively the vibrational kinetic energy

$$
\begin{aligned}
\hat{T}_{\mathrm{vib}}= & -\frac{\hbar^{2}}{2 \sqrt{w r}}\left\{\frac { 1 } { \beta ^ { 4 } } \left[\frac{\partial}{\partial \beta} \sqrt{\frac{r}{w}} \beta^{4} B_{\gamma \gamma}\right.\right. \\
& \left.-\frac{\partial}{\partial \beta} \sqrt{\frac{r}{w}} \beta^{3} B_{\beta \gamma} \frac{\partial}{\partial \gamma}\right]+\frac{1}{\beta \sin 3 \gamma}\left[-\frac{\partial}{\partial \gamma}\right. \\
& \left.\left.\times \sqrt{\frac{r}{w}} \sin 3 \gamma B_{\beta \gamma} \frac{\partial}{\partial \beta}+\frac{1}{\beta} \frac{\partial}{\partial \gamma} \sqrt{\frac{r}{w}} \sin 3 \gamma B_{\beta \beta} \frac{\partial}{\partial \gamma}\right]\right\},
\end{aligned}
$$

the rotational kinetic energy

$$
\hat{T}_{\text {rot }}=\frac{1}{2} \sum_{k=1}^{3} \frac{\hat{J}_{k}^{2}}{\mathcal{I}_{k}}
$$

and the collective potential $V_{\text {coll }} . \hat{J}_{k}(k=1,2,3$,$) denote$ the components of the angular momentum in the bodyfixed frame of a nucleus, and the mass parameters $B_{\beta \beta}$, $B_{\beta \gamma}, B_{\gamma \gamma}$, as well as the moments of inertia $\mathcal{I}_{k}$ depend on the quadrupole deformation variables $\beta$ and $\gamma$. Two additional quantities that appear in the $\hat{T}_{\text {vib }}$ term (2), $r=$ $B_{1} B_{2} B_{3}$ and $w=B_{\beta \beta} B_{\gamma \gamma}-B_{\beta \gamma}^{2}$, determine the volume element in the collective space.

The eigenvalue problem of the Hamiltonian (11) is solved using an expansion of eigenfunctions in terms of a complete set of basis functions that depend on the five collective coordinates $\beta, \gamma$ and $\Omega(\phi, \theta, \psi)$ [23]. Using the obtained collective wave functions

$$
\Psi_{\alpha}^{I M}(\beta, \gamma, \Omega)=\sum_{K \in \Delta I} \psi_{\alpha K}^{I}(\beta, \gamma) \Phi_{M K}^{I}(\Omega),
$$

various observables such as electric quadrupole transition probabilities can be calculated,

$$
B\left(E 2 ; \alpha I \rightarrow \alpha^{\prime} I^{\prime}\right)=\frac{1}{2 I+1}\left|\left\langle\alpha^{\prime} I^{\prime}|| \hat{M}(E 2) \| \alpha I\right\rangle\right|^{2},
$$

where $\hat{M}(E 2)$ is the electric quadrupole operator 23.

In the framework of 5DCH-CDFT, the microscopic collective parameters of $5 \mathrm{DCH}$ are all determined from the CDFT, which include the mass parameters $B_{\beta \beta}, B_{\beta \gamma}$, and $B_{\gamma \gamma}$, the moments of inertia $\mathcal{I}_{k}$, and the collective potential $V_{\text {coll }}$. The moments of inertia are calculated with the Inglis-Belyaev formula and the mass parameters from the cranking approximation. $V_{\text {coll }}$ is obtained by subtracting the zero-point energy corrections from the total energy d that corresponds to the solution of constrained triaxial CDFT. Detailed formalism can be found in Ref. 23.

\section{Results and discussion}

The main purpose of this study is a microscopic analysis for the collective potential energy surfaces, low-energy collective spectra, relative $B(E 2)$ transition ratios etc., 


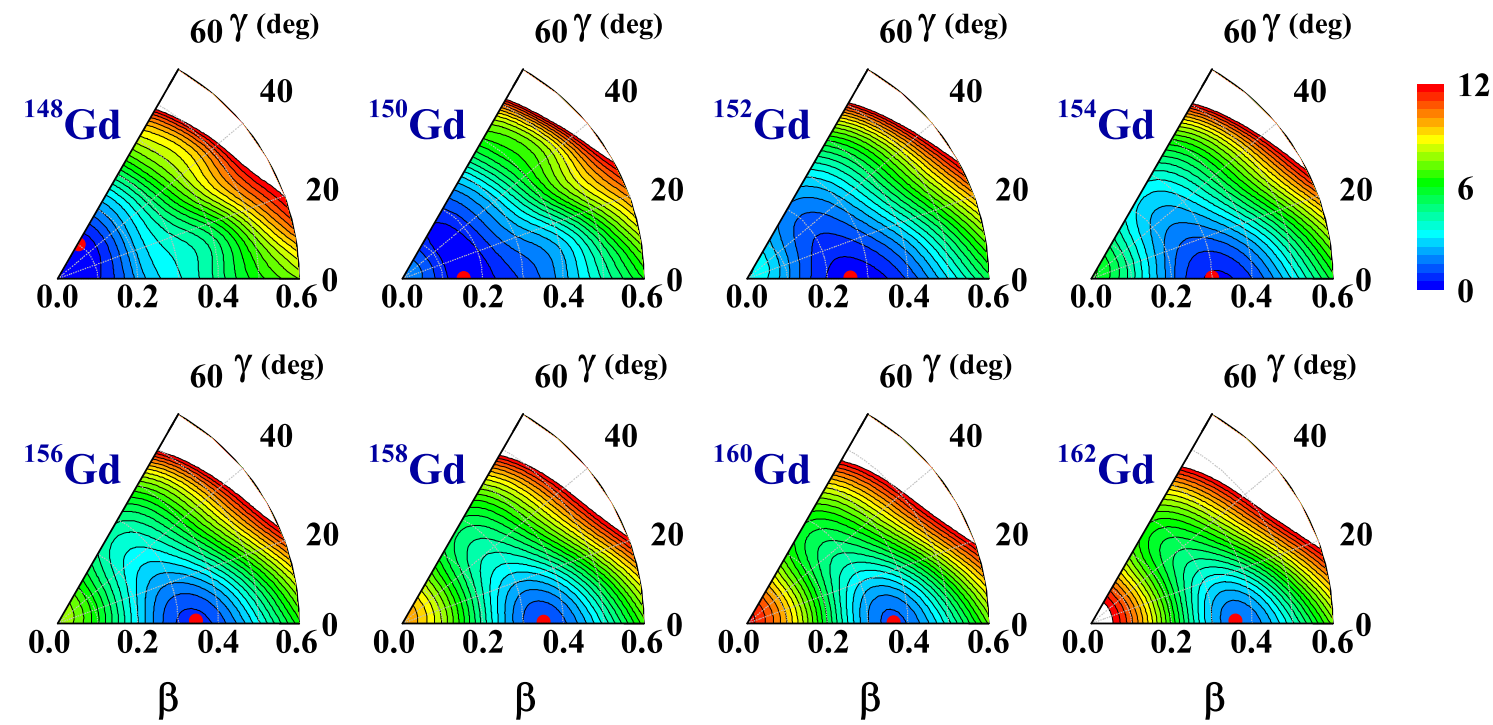

Fig. 1. (Color online) The potential energy surfaces of even-even ${ }^{148-162} \mathrm{Gd}$ isotopes in the $\beta-\gamma$ planes calculated by constrained triaxial covariant density functional theory with PC-PK1 [4]. All energies are normalized with respect to the binding energy of the absolute minimum (labeled by a red dot). The energy difference between the neighbouring contour lines is $0.5 \mathrm{MeV}$.

in the even ${ }^{148-162} \mathrm{Gd}$ isotopes using the CDFT-based $5 \mathrm{DCH}$ model. To determine the collective parameters for the 5DCH, we perform a constrained RMF plus BCS (RMF+ calculation, with the effective interaction in the particlehole channel defined by the point-coupling density functional PC-PK1 40, and a density-independent $\delta$-force in the particle-particle channel. The strength parameter of the $\delta$-force is $349.5 \mathrm{MeV} \mathrm{fm}^{3}\left(330.0 \mathrm{MeV} \mathrm{fm}^{3}\right)$ for neutron (proton), which is determined by fitting the empirical neutron (proton) pairing gap 40. The Dirac equation is solved by expanding the Dirac spinor in terms of the three dimensional harmonic oscillator basis with 14 major shells.

\subsection{Potential energy surfaces}

Firstly, to get a comprehensive understanding of the shape evolution in Gd isotopes, the PESs of even-even ${ }^{148-162} \mathrm{Gd}$ isotopes, calculated by constrained triaxial CDFT with the PC-PK1 [4] density functional are shown in Fig. 1] in the $\beta-\gamma$ planes.

For ${ }^{148} \mathrm{Gd}$, the minimum of the PES locates at $\beta=0.1$ and $\gamma=60^{\circ}$, indicating that the ground state of ${ }^{148} \mathrm{Gd}$ is of slight oblate deformation. Meanwhile, the potential energy in the region of $|\beta| \leq 0.1$ exhibits a rather weak dependence on both $\beta$ and $\gamma$. With two more neutrons, the ground state of ${ }^{150} \mathrm{Gd}$ is prolate with $\beta=0.15$, and its PES around the ground state is extremely flat in the $\gamma$ direction, where a tunnel connecting the prolate and oblate shapes can be seen. With further more neutrons, the quadrupole deformation $\beta$ of $\mathrm{Gd}$ isotopes increases from $\beta=0.15\left({ }^{150} \mathrm{Gd}\right)$ to $0.25\left({ }^{152} \mathrm{Gd}\right)$, to $0.3\left({ }^{154} \mathrm{Gd}\right)$, and to $0.35\left({ }^{156} \mathrm{Gd}\right)$, with the triaxial deformation parameter keeping at $\gamma=0^{\circ}$. Continuing to add neutrons, the deformations of the ground states of ${ }^{158,160,162} \mathrm{Gd}$ become the same as that of ${ }^{156} \mathrm{Gd}$, i.e., $\beta=0.35$ and $\gamma=0^{\circ}$. It is noted that the potential rigidness along both $\beta$ and $\gamma$ Bresctions become more and more rigid with the increase of the neutron number.

Thus, a clear shape evolution from weakly deformed $\left({ }^{148,150} \mathrm{Gd}\right)$ to soft prolate $\left({ }^{152,154} \mathrm{Gd}\right)$ and to the well deformed prolate $\left({ }^{156-162} \mathrm{Gd}\right)$ for the even-even ${ }^{148-162} \mathrm{Gd}$ isotopes is presented.

The PESs shown in Fig. 1, including the locations of the minima, are consistent with the calculations with PCF1 parameter 23, except that the PESs with PC-PK1 parameter are slightly rigid in the $\gamma$ direction. In addition, the PES of ${ }^{154} \mathrm{Gd}$ in the present calculation does not exhibit the flat behavior. This is consistent with the previous mean-field calculations $[4,5,6]$.

\subsection{Collective energy spectra}

With the collective parameters determined from CDFT, the excitation energies and the collective wave functions for each value of the total angular momentum $I$ can be obtained by diagonalizing the 5DCH Hamiltonian. As discussed in Ref. [23, in the 5DCH results, the $\gamma$ and $\beta$ bands in the deformed and transitional nuclei are usually assigned according to the distribution of the angular momentum projection $K$. That is the excited states with predominant $K=2$ components in the wave function are assigned to be $\gamma$ band, whereas the states above the yrast sequence characterized by dominant $K=0$ components are assigned to be $\beta$ band. In Figs. 2, 3, and 5, the low-lying excitation energy spectra for the ground state bands, $\gamma$ bands and $\beta$ bands of Gd isotopes calculated by $5 \mathrm{DCH}$ are displayed, respectively. The available experimental data 42 are also shown for comparison. 

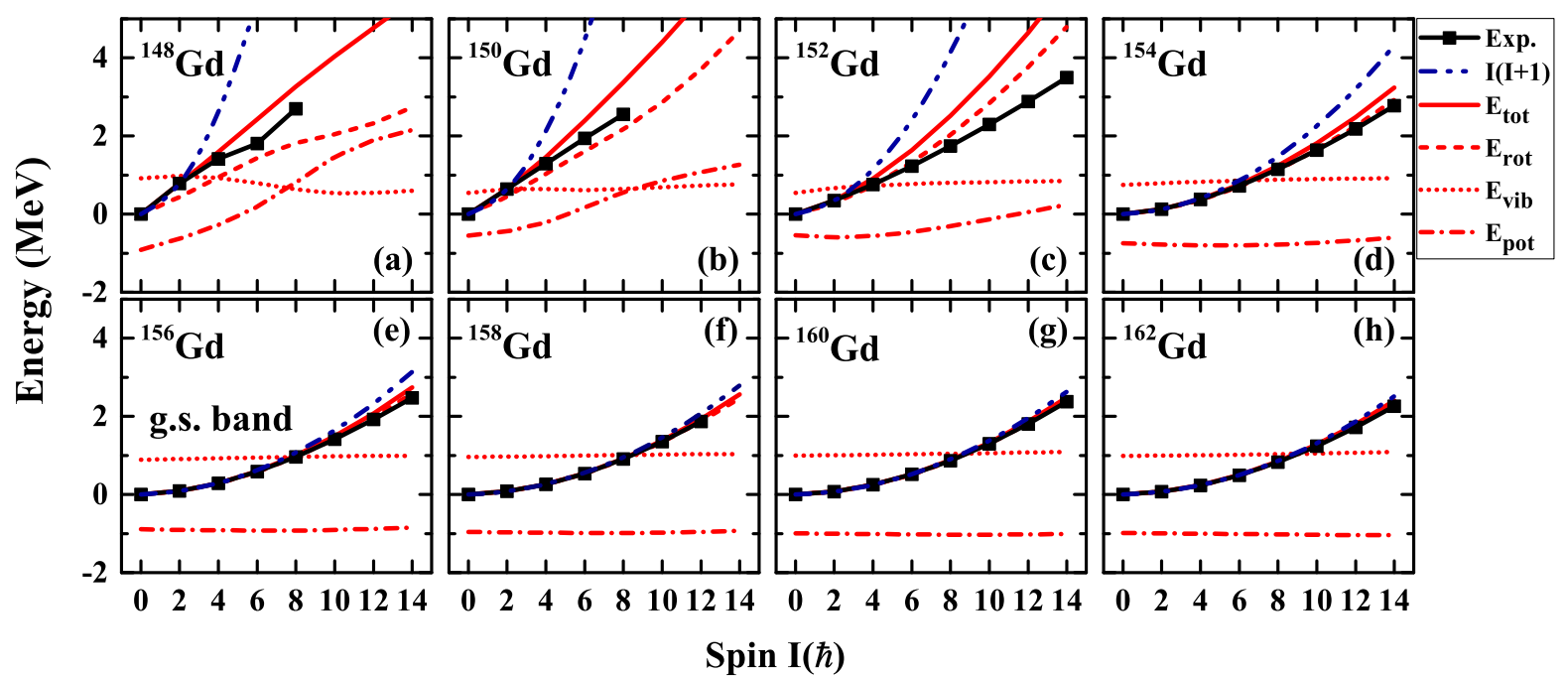

Fig. 2. (Color online) The energy spectra for ground state bands in even-even ${ }^{148-162}$ Gd isotopes calculated by $5 \mathrm{DCH}$ model based on CDFT in comparison with those available experimental data. The theoretical spectra $E_{\text {tot }}$ are normalized to the experimental energy of $2_{1}^{+} . E_{\mathrm{rot}}, E_{\mathrm{vib}}$ and $E_{\mathrm{pot}}$ represent the energy contributions from the rotational, vibrational and collective potential energy terms in the collective Hamiltonian. All the experimental data are taken from NNDC [42].

It is noted that currently, the inertia parameters are calculated by the Inglis-Belyaev formula, which does not include the Thouless-Valatin dynamical rearrangement contributions and, thus would systematically underestimate the empirical values remarkably. As illustrated in Ref. [4], the Thouless-Valatin corrections are almost independent of deformation and for a given nucleus the effective moment of inertia used in the collective Hamiltonian can simply be obtained by renormalizing the Inglis-Belyaev values by a constant factor. In the practical calculation, the theoretical result of the $2_{1}^{+}$state is normalized to the corresponding experimental $2_{1}^{+}$energy. For the even-even Gd isotopes from ${ }^{148} \mathrm{Gd}$ to ${ }^{162} \mathrm{Gd}$, the factors are $1.889,0.863$, $0.818,1.379,1.500,1.500,1.455$ and 1.411 , respectively.

In Fig. 2 the energies of the ground-state (g.s.) bands in Gd isotopes obtained from 5DCH calculations are compared with the available experimental data. The energy contribution from each term of the collective Hamiltonian, i.e., the rotational, vibrational and collective potential energy terms are also present, which are calculated through the following form

$$
\begin{aligned}
& E_{\text {rot }}=\left\langle\Psi_{\alpha}^{I}\left|\hat{T}_{\text {rot }}\right| \Psi_{\alpha}^{I}\right\rangle, \\
& E_{\text {vib }}=\left\langle\Psi_{\alpha}^{I}\left|\hat{T}_{\mathrm{vib}}\right| \Psi_{\alpha}^{I}\right\rangle, \\
& E_{\mathrm{pot}}=\left\langle\Psi_{\alpha}^{I}\left|V_{\mathrm{coll}}\right| \Psi_{\alpha}^{I}\right\rangle .
\end{aligned}
$$

In Fig. 2, to illustrate the deviation of $E_{\text {tot }}$ from the ideal SU(3) character, a line calculated by $c * I(I+1)$ form is also included, in which the coefficient $c$ is determined by the $E\left(2_{1}^{+}\right)$. As shown in Fig. 2. the agreements between the theoretical energy spectra and the experimental data for Gd isotopes are overall good. For the light ${ }^{148,150} \mathrm{Gd}$ isotopes, the theoretical results predict larger energy spacing. It is noted that in the experimental ground state band of ${ }^{148} \mathrm{Gd}$, the energy spacing decreases with spin when $I \leq 6 \hbar$. As indicated in Refs. 44, 45, the yrast $0^{+}, 2^{+}$,
$4^{+}$, and $6^{+}$states in ${ }^{148} \mathrm{Gd}$ are formed by the coupling of the two $1 f_{7 / 2}$ neutron, which does not belong to the collective excitation and is beyond the scope of our description. Besides, ${ }^{148} \mathrm{Gd}$ is almost a doubly magic nucleus, in which the neutron number is close to the magic number $N=82$ and the proton number $Z=64$ is also sometimes regarded as a kind of magic number, and this is the main reason why the shell-model approach gives better results. The energy spectra for both the experimental and theoretical ground state bands deviate significantly from the ideal rigid rotor. The energy contributions $E_{\mathrm{rot}}, E_{\mathrm{vib}}$ and $E_{\mathrm{pot}}$ indicate that the increase of energy with spin comes from both the rotational and collective potential terms. This could be understood that due to the soft PES, the deformation of ${ }^{148,150} \mathrm{Gd}$ will increase significantly with the increase of angular momentum, which is similar to the picture of 'tidal wave' 35 . For ${ }^{152,154} \mathrm{Gd}$, the theoretical results overestimate the experimental data a little. However, the trends of the level spacing increasing slightly with spin in the experimental ground state bands are still reproduced by the calculations. For ${ }^{156-162} \mathrm{Gd}$, the experimental data are well reproduced by the 5DCH calculations, both of the experimental data and theoretical values obey the rule of $I(I+1)$ approximately, indicating that the ground state bands have the $\mathrm{SU}(3)$ character. Due to the stable shapes of ${ }^{156-162} \mathrm{Gd}$, the increase of the ground state bands energies all come from the rotational term, while $E_{\mathrm{vib}}$ and $E_{\text {pot }}$ keep constant with the increase of spin, as can be seen in Fig. 2.

For the $\gamma$ bands shown in Fig. 3, the agreements between the theoretical results and available experimental data are good. For ${ }^{152} \mathrm{Gd}$, the energy difference of the bandhead between the experimental and theoretical values is about $350 \mathrm{keV}$, and for all the other nuclei the differences are smaller than $150 \mathrm{keV}$. The increasing behaviors for the $\gamma$ bands in ${ }^{156-162} \mathrm{Gd}$ are consistent with the 

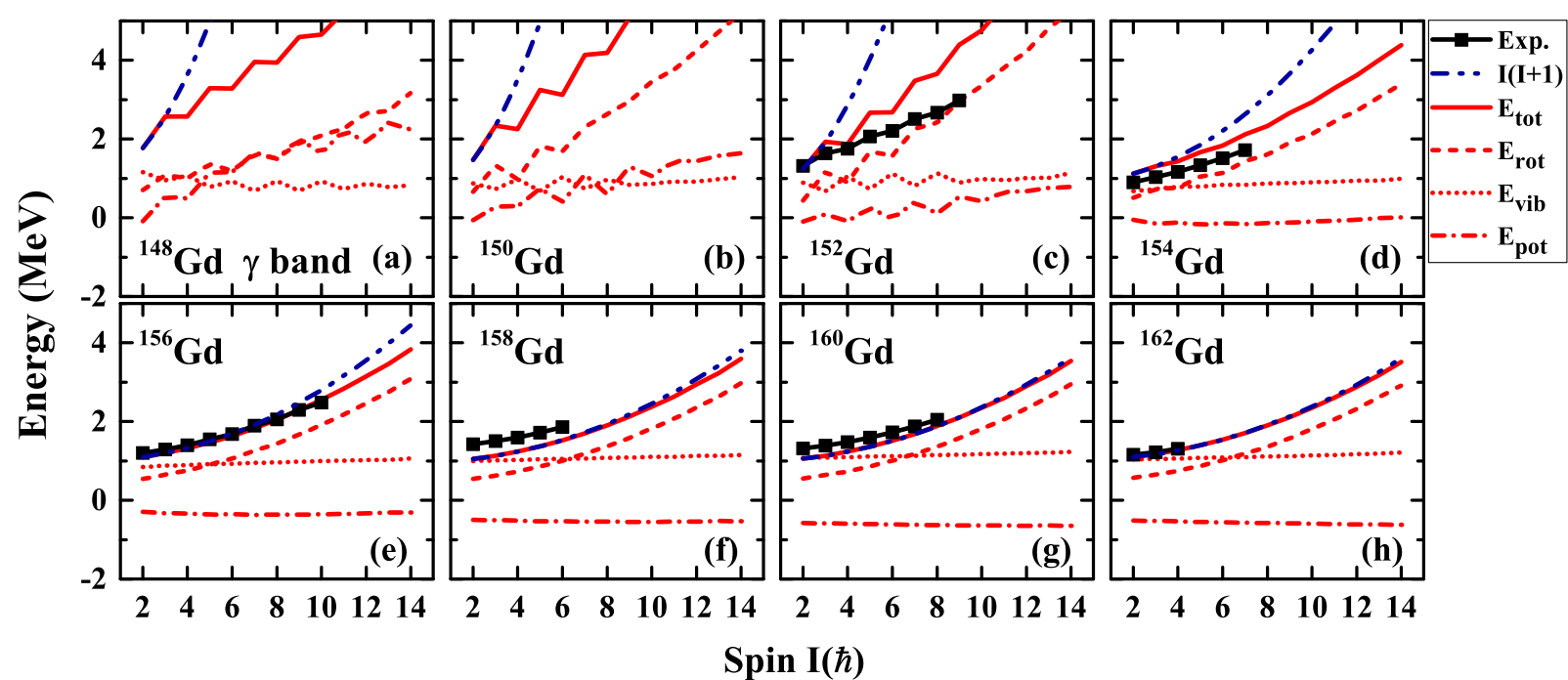

Fig. 3. (Color online) The energy spectra for $\gamma$ bands in even-even ${ }^{148-162} \mathrm{Gd}$ isotopes calculated by $5 \mathrm{DCH}$ model based on CDFT in comparison with those available experimental data. $E_{\mathrm{rot}}, E_{\mathrm{vib}}$ and $E_{\mathrm{pot}}$ represent the energy contributions from the rotational, vibrational and collective potential energy terms in the collective Hamiltonian. All the experimental data are taken from NNDC 42 .

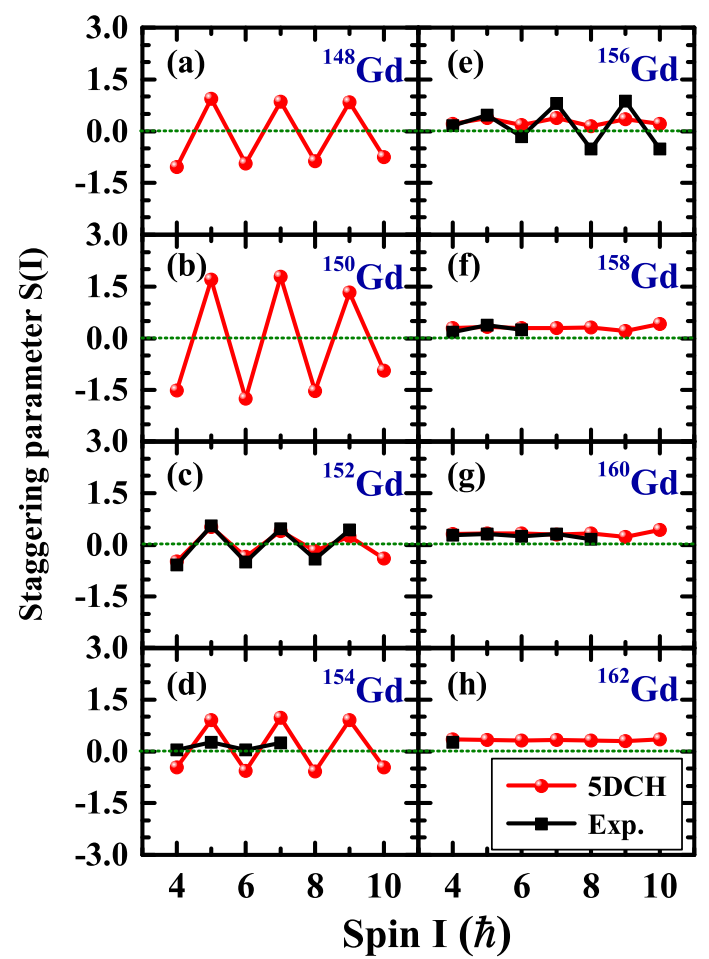

Fig. 4. (Color online) The staggering parameter $S(I)$ of eveneven ${ }^{148-162} \mathrm{Gd}$ isotopes calculated by 5DCH-CDFT in comparison with the experimental data 42 .

$I(I+1)$ lines, indicating that the $\mathrm{SU}(3)$ symmetries are still conserved.

The $\gamma$ band staggering parameter

$$
S(I)=\frac{[E(I)-E(I-1)]-[E(I-1)-E(I-2)]}{E\left(2_{1}^{+}\right)}
$$

is an indicator of the triaxial softness/rigidness [46 47. For a vibrator, the $S(I)$ oscillates between negative values for even-spin states and positive values for odd-spin states, with the magnitude keeps at $|S(I)=1|$. For a nucleus with a deformed $\gamma$-soft potential, the $S(I)$ oscillates between negative values for even-spin states and positive values for odd-spin states, with the magnitude slowly increasing with spin. In the limit of an axially symmetric rotor, the $S(I)$ values are positive, small (0.33), and constant as a function of spin.

In Fig. 4, we plot the theoretical staggering parameters $S(I)$ for the $\gamma$ bands in Gd isotopes, in comparison with the available experimental data. Except ${ }^{154} \mathrm{Gd}$ and ${ }^{156} \mathrm{Gd}$, the experimental staggering parameters are well reproduced by the $5 \mathrm{DCH}$ calculations. For ${ }^{148} \mathrm{Gd}$, the $S(I)$ parameters are negative for even-spin states and positive for odd-spin states and the magnitude of $S(I)$ keeping about one, indicating that ${ }^{148} \mathrm{Gd}$ possesses the spherical vibrator behavior. For ${ }^{150,152,154} \mathrm{Gd}$, the theoretical $S(I)$ parameters are negative for even-spin states and positive for odd-spin states, close to that of a $\gamma$ soft potential, and is in consistent with the $\gamma$ soft PESs shown in Fig. 1, For ${ }^{156-162} \mathrm{Gd}$, the theoretical $S(I)$ parameters are close to a constant value of 0.33 , indicating the axial rotor character. The difference between the theoretical values and experimental data in ${ }^{154} \mathrm{Gd}$ may be due to the too soft potential predicted by CDFT, while the difference in ${ }^{156} \mathrm{Gd}$ may be due to the too rigid potential predicted by CDFT.

The rotational band built on the $0_{2}^{+}$state in deformed rare-earth nuclei has been usually labelled as $\beta$-band for many years. In the present work, we also follow such convention. But it is worth mentioning that the nature of the lowest-lying excited $0_{2}^{+}$state is still debated heavily. The investigation by Garrett et al. shows that most of the $0_{2}^{+}$ states are not $\beta$ vibrations [48,49]. For the $\beta$ bands, as can be seen in Fig. 5. the calculations do not reproduce the 

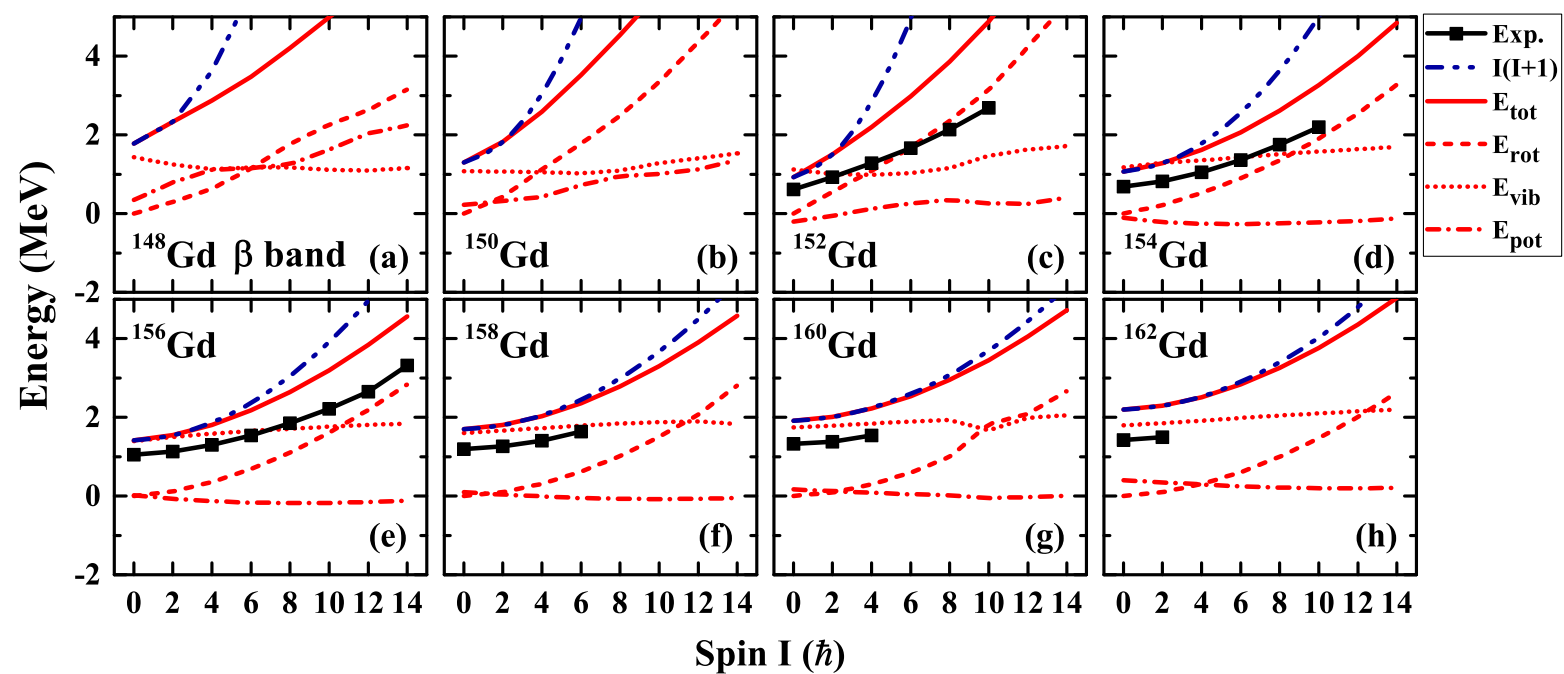

Spin I $(\hbar)$

Fig. 5. (Color online) The energy spectra for $\beta$ bands in even-even ${ }^{148-162}$ Gd isotopes calculated by $5 \mathrm{DCH}$ model based on CDFT in comparison with those available experimental data. $E_{\mathrm{rot}}, E_{\mathrm{vib}}$ and $E_{\mathrm{pot}}$ represent the energy contributions from the rotational, vibrational and collective potential energy terms in the collective Hamiltonian. All the experimental data are taken from NNDC [42].

data as well as the ground state bands and the $\gamma$ bands. The calculated bandhead energies of $\beta$ band $0_{2}^{+}$are always higher than the data. This could be understood by the fact that the bandhead energy of the $\beta$ band depends on the mass parameters rather sensitively. In the present calculations, the mass parameters are underestimated due to the absence of Thouless-Valatin dynamical rearrangement contributions. One may adjust the mass parameters to reproduce the experimental values. However, the mass parameters are so complicated, that there are no simple estimates of the Thouless-Valatin corrections can be obtained [50. Furthermore, the current model does not contain the coupling of nuclear shape oscillations with pairing vibrations 51 . The excitation energy of the $0^{+}$is also very sensitive to such coupling 27. Here, we would like to mention that, the energies of the ground state band and $\gamma$ band do not evidently depend on the mass parameters. Therefore, despite the lack of pairing vibration coupling in the mass parameters, the g.s. and $\gamma$ bands can still be well reproduced. Although the $\beta$ band energies are overestimated by the calculations, the experimental feature for the $\beta$ bands can be still reproduced. That is the experimental and theoretical $\beta$ bands both behave as rotational bands.

For ${ }^{156-162} \mathrm{Gd}$, the $\mathrm{SU}(3)$ characters for the $\beta$ bands are a little different from those in the g.s. and $\gamma$ bands. Generally, the $E_{\text {tot }}$ of $\beta$ bands in the ${ }^{156-162} \mathrm{Gd}$ are consistent with the $I(I+1)$ lines in the region of low angular momentum. With the increase of the angular momentum, the deviations of $E_{\text {tot }}$ from $I(I+1)$ become more and more apparent. This indicates the break of SU(3) symmetries in the $\beta$ bands. The deviations with increasing angular momentum become smaller when the neutron number increases. The increase of the total energies comes from the nuclear rotation, while the energy contributions coming from the vibration and collective Hamiltonian re-

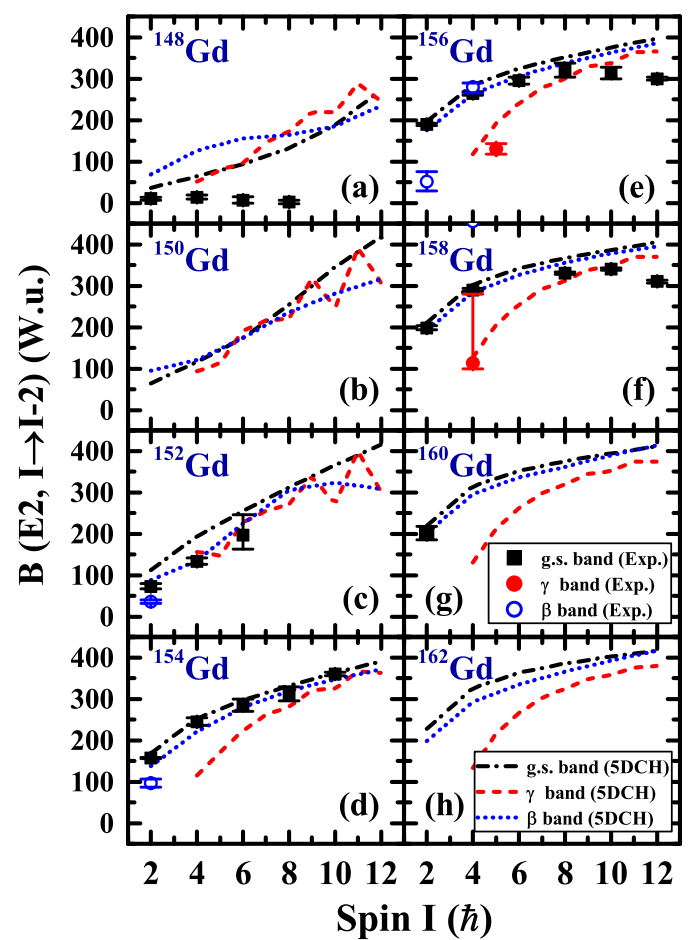

Fig. 6. (Color online) The intraband $B(E 2 ; I \rightarrow I-2)$ values (in Weisskopf unit) for the ground-state, $\gamma$ and $\beta$ bands in ${ }^{148-162} \mathrm{Gd}$. Theoretical values calculated with the PC-PK1 relativistic density functional are compared with the data 42 .

main almost unchanged, due to the well-deformed shapes of ${ }^{156-162} \mathrm{Gd}$ as shown in Fig. 1]

For the energy spectra obtained from PC-F1, the increasing trend with spin for the g.s., $\gamma$ and $\beta$ bands are similar to those from PC-PK1, but the bandhead energies for the $\beta$ bands from PC-F1 are about $250 \mathrm{keV}$ lower 
for ${ }^{156-160} \mathrm{Gd}$, due to the softer PESs predicted by PCF1 [23].

\section{3 $B(E 2)$ transition strength}

The intraband $B(E 2)$ transition strength in the g.s., $\gamma$ and $\beta$ bands are also shown in comparison with the available data in Fig. 6 For ${ }^{148} \mathrm{Gd}$, the 5DCH calculation overestimates the data significantly. This may be because that the excitation mode in ${ }^{148} \mathrm{Gd}$ does not belong to the collective excitation, as indicated in Refs. 44, 45, the yrast $0^{+}, 2^{+}, 4^{+}$, and $6^{+}$states are formed by the coupling of the two $1 f_{7 / 2}$ neutron. The available experimental data in ${ }^{152-162} \mathrm{Gd}$ are well reproduced by the $5 \mathrm{DCH}$ calculations. It is also found that in ${ }^{154-162} \mathrm{Gd}$, the $B(E 2)$ transitions of the g.s. and $\beta$ bands are similar, and the $B(E 2)$ values in the $\gamma$ bands are smaller than those of the g.s. and $\beta$ bands. In addition, the increasing behaviors for the $B(E 2)$ of each band in ${ }^{156-162} \mathrm{Gd}$ are very similar, indicating similar structures in these four nuclei.

The relative $B(E 2)$ ratios from IBM correspond to parameter-free SU(3) PDS calculations and therefore they are ideal quantities to decide about the validity or not of the concept of SU(3) PDS for a given nucleus or model. Fig. 7 presents the comparison of the relative $B(E 2)$ ratios calculated by $5 \mathrm{DCH}$ with the available IBM results 12 and experimental data [42,12 for Gd isotopes. The theoretical $R_{4 / 2}$ ratios $\left(R_{4 / 2}=E\left(4_{1}^{+}\right) / E\left(2_{1}^{+}\right)\right)$are also shown in the figure. It is found that the available experimental data display the general characteristic that the spindecreasing transitions are larger than the spin-increasing transitions for ${ }^{156-160} \mathrm{Gd}$, except for the transition from $5_{\gamma}^{+}$in ${ }^{156} \mathrm{Gd}$, which has an opposite behavior. The spinconservation transitions are the largest for transitions from even-spin states in the $\gamma$ bands. The data of ${ }^{156-160} \mathrm{Gd}$ can be well reproduced by the 5DCH theoretical calculations. For transitions from $3_{\gamma}^{+}, 4_{\gamma}^{+}$, and $5_{\gamma}^{+}$states in the transitional nucleus ${ }^{154} \mathrm{Gd}$, the spin-increasing transitions are larger than the spin-decreasing ones, and the transition from $2_{\gamma}^{+}$to $0_{1}^{+}$is larger than that to $4_{1}^{+}$. The spinconservation transitions are also the largest for the transitions from even spin states in the $\gamma$ band. The data of ${ }^{154} \mathrm{Gd}$ can be also reasonably reproduced by the $5 \mathrm{DCH}$ calculations.

For the results obtained from $5 \mathrm{DCH}$, it is clearly seen that for ${ }^{148} \mathrm{Gd}$, there only exist spin-conservation transitions from the even-spin states and spin-increasing transitions from odd-spin states in the $\gamma$ band. The transitions to other states in the g.s. band are so weak that they are not visible. This can be explained by the vibrator selection rules as emphasized in Ref. [52], that the spin-decreasing transitions from non-yrast to yrast states are forbidden due to the phonon number which can be only changed by 1 . Similar phenomena can be found in ${ }^{150} \mathrm{Gd}$. Only the transitions from the $4_{\gamma}^{+}$state in ${ }^{150} \mathrm{Gd}$ are different from those in ${ }^{148} \mathrm{Gd}$. For transitions from the $4_{\gamma}^{+}$state in ${ }^{150} \mathrm{Gd}$, the transition to $6_{1}^{+}$is the largest, and the spinconservation transition is a bit small. For ${ }^{152} \mathrm{Gd}$, the rela- tive relations for transitions from $3_{\gamma}^{+}, 4_{\gamma}^{+}$, and $5_{\gamma}^{+}$are similar to those in ${ }^{150} \mathrm{Gd}$, and the spin-increasing transitions are the largest, while the transitions from $2_{\gamma}^{+}$in ${ }^{152} \mathrm{Gd}$ are different from those in ${ }^{150} \mathrm{Gd}$. The transition from $2_{\gamma}^{+}$ to $4_{1}^{+}$is much larger than the transition to $2_{1}^{+}$, and the spin-conservation $2_{\gamma}^{+}$to $2_{1}^{+}$transition is the smallest. This behavior can be understood by the significant mixing of $K$ quantum numbers between $K=0$ and $K=2$ of the $\gamma$ bandhead for ${ }^{152} \mathrm{Gd}$. The corresponding $K$ distributions of the $\gamma$ band head are $52 \%$, and $48 \%$ for $K=0$ and $K=2$, respectively. For ${ }^{154} \mathrm{Gd}$, the $5 \mathrm{DCH}$ spin-decreasing transitions are larger than the spin-increasing ones, except for the transitions from the $5_{\gamma}^{+}$state, which have an opposite trend. The spin-conservation transitions are the largest for the even-spin states in the $\gamma$ band. The relative $B(E 2)$ transition ratios for ${ }^{156-162} \mathrm{Gd}$ are similar to those of ${ }^{154} \mathrm{Gd}$, with only small differences in the transition strength.

As shown in Fig. 7 the available IBM results show good agreements with the data. For ${ }^{158} \mathrm{Gd}$, the differences between IBM and 5DCH are smaller than those in ${ }^{154} \mathrm{Gd}$. As described in Ref. [12, PDS systematically underestimates the spin-increasing transitions and overestimates the spin-decreasing transitions in the rare-earth region. However, the discrepancies between the data and 5DCH results are not systematical. This could be explained by the fact that the $5 \mathrm{DCH}$ calculations have taken the configuration mixing into account self-consistently. Furthermore, for ${ }^{154-156} \mathrm{Gd}$, the calculated transitions from the $5_{\gamma}^{+}$states by $5 \mathrm{DCH}$ are opposite to the PDS descriptions, which predict the $5_{\gamma}^{+}$to $4_{1}^{+}$transition larger than the $5_{\gamma}^{+}$to $6_{1}^{+}$transition. However, the transitions from $5_{\gamma}^{+}$states to $6_{1}^{+}$are larger than those to $4_{1}^{+}$states calculated by $5 \mathrm{DCH}$, which are consistent with the experimental values, e.g., in ${ }^{156} \mathrm{Gd}$.

This agreement is not trivial, by including the configuration mixing effect self-consistently, the parameter-free 5 DCH calculations can not only well reproduce the data of relative $B(E 2)$ for well-deformed rotors ${ }^{156-162} \mathrm{Gd}$, but also reproduce the data for the transitional nucleus ${ }^{154} \mathrm{Gd}$, for which the SU(3) PDS is not expected to work 12 . From the 5DCH-CDFT calculations, it seems that ${ }^{156-162} \mathrm{Gd}$ may fulfil the concept of SU(3) PDS.

\subsection{Quadrupole deformation}

Experimentally, with the reduced quadrupole transition probabilities, the deformation parameter $\beta$ could be extracted 53 54. In the framework of $5 \mathrm{DCH}$, the deformation of a nucleus can be characterized by the average of the invariant $\beta^{2}, \beta^{3} \cos 3 \gamma$ and their combination. Take $\beta^{2}$ as an example, the average value of $\beta^{2}$ in the state $|\alpha I\rangle$ is

$$
\left\langle\beta^{2}\right\rangle_{I \alpha}=\left\langle\Psi_{\alpha}^{I}\left|\beta^{2}\right| \Psi_{\alpha}^{I}\right\rangle=\sum_{K \in \Delta I} \int \beta^{2}\left|\psi_{\alpha, K}^{I}(\beta, \gamma)\right|^{2} d \tau_{0} .
$$

The expectation values of the quadrupole deformation $\langle\beta\rangle$ and $\langle\gamma\rangle$ together with their fluctuations $\Delta \beta$ and $\Delta \gamma$ for 


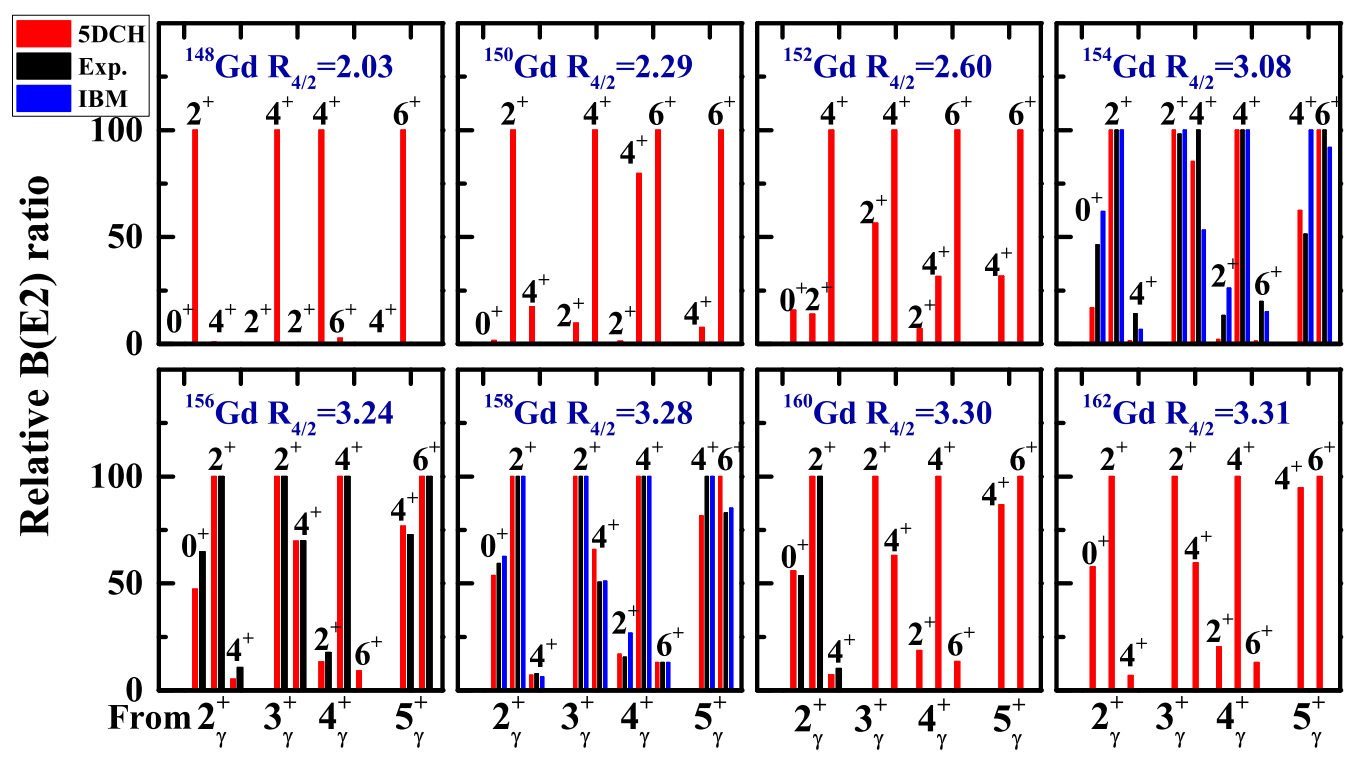

Fig. 7. (Color online) Comparison of $5 \mathrm{DCH}$ predictions with the data on the relative $\gamma$ band to ground state band $E 2$ transitions in even-even ${ }^{148-162} \mathrm{Gd}$ isotopes. The red (black) bar are the 5DCH predictions (data). The available IBM results 12 (blue bar) are also shown for comparison. The largest transition is taken as reference and normalized to 100 for each initial state. The $R_{4 / 2}$ values obtained by $5 \mathrm{DCH}$ are also given in the figure.

each given state can be obtained 23

$$
\begin{aligned}
& \langle\beta\rangle=\sqrt{\left\langle\beta^{2}\right\rangle} \\
& \langle\gamma\rangle=\arccos \left(\left\langle\beta^{3} \cos 3 \gamma\right\rangle / \sqrt{\left\langle\beta^{4}\right\rangle\left\langle\beta^{2}\right\rangle}\right) / 3
\end{aligned}
$$

and

$$
\begin{aligned}
& \Delta \beta=\frac{\sqrt{\left\langle\beta^{4}\right\rangle-\left\langle\beta^{2}\right\rangle^{2}}}{2\langle\beta\rangle}, \\
& \Delta \gamma=\frac{1}{3 \sin 3\langle\gamma\rangle} \sqrt{\frac{\left\langle\beta^{6} \cos ^{2} 3 \gamma\right\rangle}{\left\langle\beta^{6}\right\rangle}-\frac{\left\langle\beta^{3} \cos 3 \gamma\right\rangle^{2}}{\left\langle\beta^{4}\right\rangle\left\langle\beta^{2}\right\rangle} .}
\end{aligned}
$$

In Fig. 8, the obtained results from 5DCH calculations for the ground states of $\mathrm{Gd}$ isotopes are presented. For a comparison, the available experimental data 42 of $\beta$ deformation are also shown in Fig. 8(a). It can be clearly seen from Fig. 8 (a) that with increasing mass number, the $\langle\beta\rangle$ becomes larger and larger. This is consistent with the results of CDFT as shown in Fig. 1] where the $\beta$ values of the minimal of the PES also increase as the mass number increases. The available experimental data increase with mass number. Clearly, the $5 \mathrm{DCH}$ calculations reproduce the data rather well. For ${ }^{148,152} \mathrm{Gd}$, the data are a little overestimated.

The trend of $\langle\gamma\rangle$ is opposite to that of $\langle\beta\rangle$, i.e., decreases with mass number. The $\langle\gamma\rangle$ starts from nearly $30^{\circ}$ for ${ }^{148} \mathrm{Gd}$ to $10^{\circ}$ for ${ }^{162} \mathrm{Gd}$. It is noted that the $\langle\gamma\rangle$ differences among ${ }^{154-162} \mathrm{Gd}$ are quite small, which means that $\langle\gamma\rangle$ has reached a stable value, and the triaxiality of $\mathrm{Gd}$ isotopes becomes stable against the neutron number. Similar phenomena can also be found in the PES figures. The trends of $\Delta \beta$ and $\Delta \gamma$ for Gd isotopes are similar. The shape fluctuation increases with mass number at first up to ${ }^{152} \mathrm{Gd}$, then begins to decrease. This indicates that

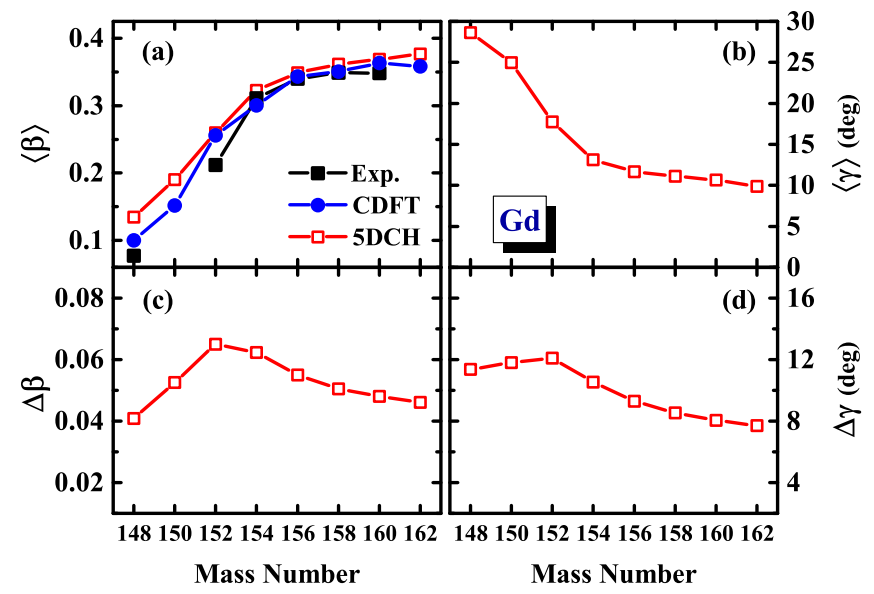

Fig. 8. (Color online) The deformation parameters $\langle\beta\rangle$ and $\langle\gamma\rangle$ as well as the corresponding fluctuations $\Delta \beta$ and $\Delta \gamma$ of $0_{1}^{+}$state in even-even ${ }^{148-162} \mathrm{Gd}$ isotopes calculated by $5 \mathrm{DCH}$ model based on CDFT. The experimental data 42] (black cubic) are also presented.

${ }^{152} \mathrm{Gd}$ is a transitional nucleus in the even-even ${ }^{148-162} \mathrm{Gd}$ isotopes.

In Fig. 9. taking ${ }^{148,152,156,160} \mathrm{Gd}$ as examples, we plot the $\langle\beta\rangle$ and $\langle\gamma\rangle$ together with the fluctuations of the quadrupole deformations $\Delta \beta$ and $\Delta \gamma$, as functions of spin for the ground state bands. For ${ }^{148} \mathrm{Gd}$, the $\langle\beta\rangle$ value increases with spin, while the $\langle\gamma\rangle$ value decreases with spin rapidly. For its quadrupole fluctuations $\Delta \beta$ and $\Delta \gamma$, they exhibit firstly increasing and then decreasing trends. The expectation values of $\langle\beta\rangle$ and $\langle\gamma\rangle$ for ${ }^{152} \mathrm{Gd}$ are similar to those for ${ }^{148} \mathrm{Gd}$, but with larger a initial value $\langle\beta\rangle=0.26$ and smaller initial value $\langle\gamma\rangle=18^{\circ}$. With the increase of spin, 


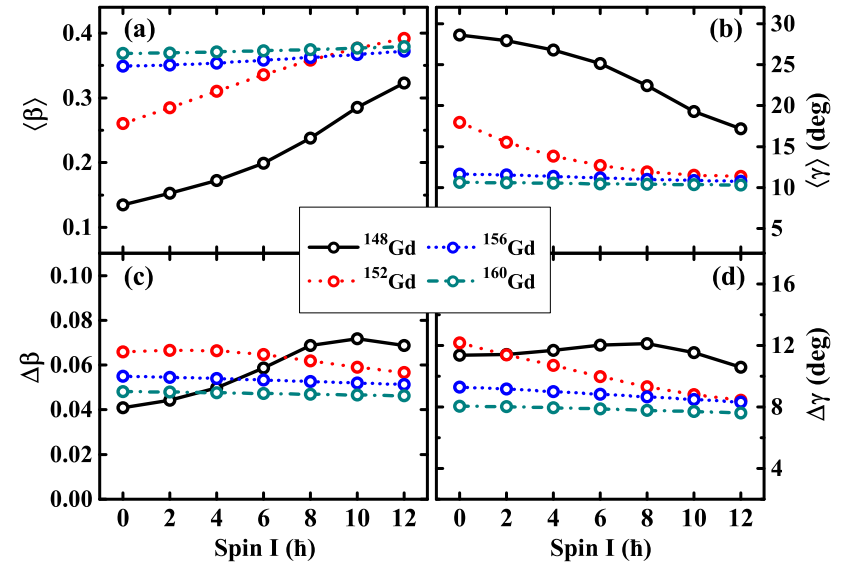

Fig. 9. (Color online) The deformation parameters $\langle\beta\rangle$ and $\langle\gamma\rangle$ as well as the corresponding fluctuations $\Delta \beta$ and $\Delta \gamma$ of ground state bands in even-even ${ }^{148,152,156,160} \mathrm{Gd}$ isotopes as functions of spin calculated by $5 \mathrm{DCH}-\mathrm{CDFT}$.

$\langle\beta\rangle$ will keep increasing while $\langle\gamma\rangle$ will finally reach a stable value of $\langle\gamma\rangle \sim 12^{\circ}$. The strong dependence on the spin of the deformations presented here by ${ }^{148,152} \mathrm{Gd}$ is associated to its PES, shown in Fig. 1, where the soft behavior can be clearly seen. For ${ }^{156,160} \mathrm{Gd}$, all of the four quantities $\langle\beta\rangle,\langle\gamma\rangle, \Delta \beta$, and $\Delta \gamma$ are almost independent of spin. This indicates that the shapes of ${ }^{156,160} \mathrm{Gd}$ are so stable that their deformations do not change with spin. Thus, the energy spectra for ${ }^{156,160} \mathrm{Gd}$ shown in Figs. 2, 3, and 5 exhibit the rigid rotor behaviors.

\subsection{Single particle levels}

To investigate the shape evolution in Gd isotopes microscopically, the neutron and proton single-particle levels as functions of the quadrupole deformation $\beta$ in $\mathrm{Gd}$ isotopes obtained from the CDFT are illustrated. As the single particle levels for the $\mathrm{Gd}$ isotopes are similar, here, we only take ${ }^{156} \mathrm{Gd}$ as an example, as shown in Fig. 10. The deformations of the ground states in Gd isotopes are also marked in the last occupied levels.

For ${ }^{148} \mathrm{Gd}$, which possesses a near spherical oblate shape, the last neutron occupies the $1 h_{9 / 2}$ orbital and the levels around the minimum are very dense in both neutron and proton single particle levels, which means that the configuration is easy to change. This corresponds to the soft character around $\beta \sim 0.1$ of ${ }^{148} \mathrm{Gd}$ as shown in Fig. 1, For ${ }^{150-152} \mathrm{Gd}$, the last neutron still occupies the $1 h_{9 / 2}$ orbital, while the level densities around the minimum point become less dense than ${ }^{148} \mathrm{Gd}$. As a result, the PESs become a bit more rigid. With two more neutrons, the orbital occupied by the last neutron in ${ }^{154} \mathrm{Gd}$ changes to the $1 i_{13 / 2}$. As an intruder orbital, the interaction between the $1 i_{13 / 2}$ and the adjacent negative parity $1 h_{9 / 2}$ levels is very weak, therefore, the ground state configuration of ${ }^{154} \mathrm{Gd}$ becomes relatively stable. With the increase of neutron numbers, more neutrons occupy the $1 i_{13 / 2}$. Thus, the ground state configurations of ${ }^{156-162} \mathrm{Gd}$ are difficult to change. Be- sides, a clear energy gap could be seen around the last occupied levels in the ${ }^{156-162} \mathrm{Gd}$. As a result, the PESs of ${ }^{156-162} \mathrm{Gd}$ show well-deformed characters. The occupied $1 i_{13 / 2}$ levels in the ground states of Gd isotopes make the deformations so stable that even do not change with the increase of angular momentum, as shown in Fig. 9]

As for the proton single particle levels, the last occupied level is $2 d_{5 / 2}$ in ${ }^{148} \mathrm{Gd}$, and $1 h_{11 / 2}$ for all the other nuclei. Since the proton numbers for the Gd isotopes are identical, the difference of the occupation situations is caused by different neutron numbers. Therefore, the occupations of neutron $1 i_{13 / 2}$ levels, which lead to the welldeformed prolate shapes of ${ }^{156-162} \mathrm{Gd}$ and result in the rigid rotor behavior for the energy spectra, are essential for these isotopes.

\section{Summary}

In conclusion, the low-lying states for the even-even ${ }^{148-162} \mathrm{Gd}$ isotopes have been investigated in the framework of 5DCHCDFT. A clear shape evolution from weakly deformed ${ }^{148,150} \mathrm{Gd}$ to $\gamma$-soft ${ }^{152,154} \mathrm{Gd}$ to well-deformed prolate ${ }^{156-162} \mathrm{Gd}$ has been presented. The shapes of ${ }^{156-162} \mathrm{Gd}$ are all welldeformed prolate with the minima located at $\beta \sim 0.35$, and these deformations are almost independent on the angular momentum. The available experimental data are reproduced by the calculations, including the energy spectra, $\gamma$ band staggering and intraband $B(E 2)$ transition probabilities for ground-state $\gamma$ and $\beta$ bands. From the energy spectra, it is found that the $\mathrm{SU}(3)$ symmetry is conserved approximately in the ground state and $\gamma$ bands, while broken in the $\beta$ bands. Furthermore, by including the configuration mixing self-consistently, the $5 \mathrm{DCH}$ calculations can not only describe the relative $B(E 2)$ ratios for the PDS candidates ${ }^{158} \mathrm{Gd}$ well, but also reproduce the relative $B(E 2)$ ratios for transitional nucleus ${ }^{154} \mathrm{Gd}$. It is found that the occupations of neutron $1 i_{13 / 2}$ orbitals, which lead to the stable PESs of ${ }^{156-162} \mathrm{Gd}$, are essential for these isotopes.

Precious supervision from Prof. Jie Meng and fruitful discussion with Prof. Peter Ring and Dr. Pengwei Zhao are highly acknowledged. This work was partly supported by the Chinese Major State 973 Program No.2013CB834400, the National Natural Science Foundation of China (Grants No. 11375015, 11461141002, 11175002, 11335002), the Research Fund for the Doctoral Program of Higher Education (Grant No. 20110001110087), Deutsche Forschungsgemeinschaft (DFG) and National Natural Science Foundation of China (NSFC) through funds provided to the Sino-German CRC 110 "Symmetries and the Emergence of Structure in QCD" and the China Postdoctoral Science Foundation (Grant No. 2015M580007 and No. 2016T90007).

\section{References}

1. R. F. Casten and N. V. Zamfir, Phys. Rev. Lett. 87, 052503 (2001). 

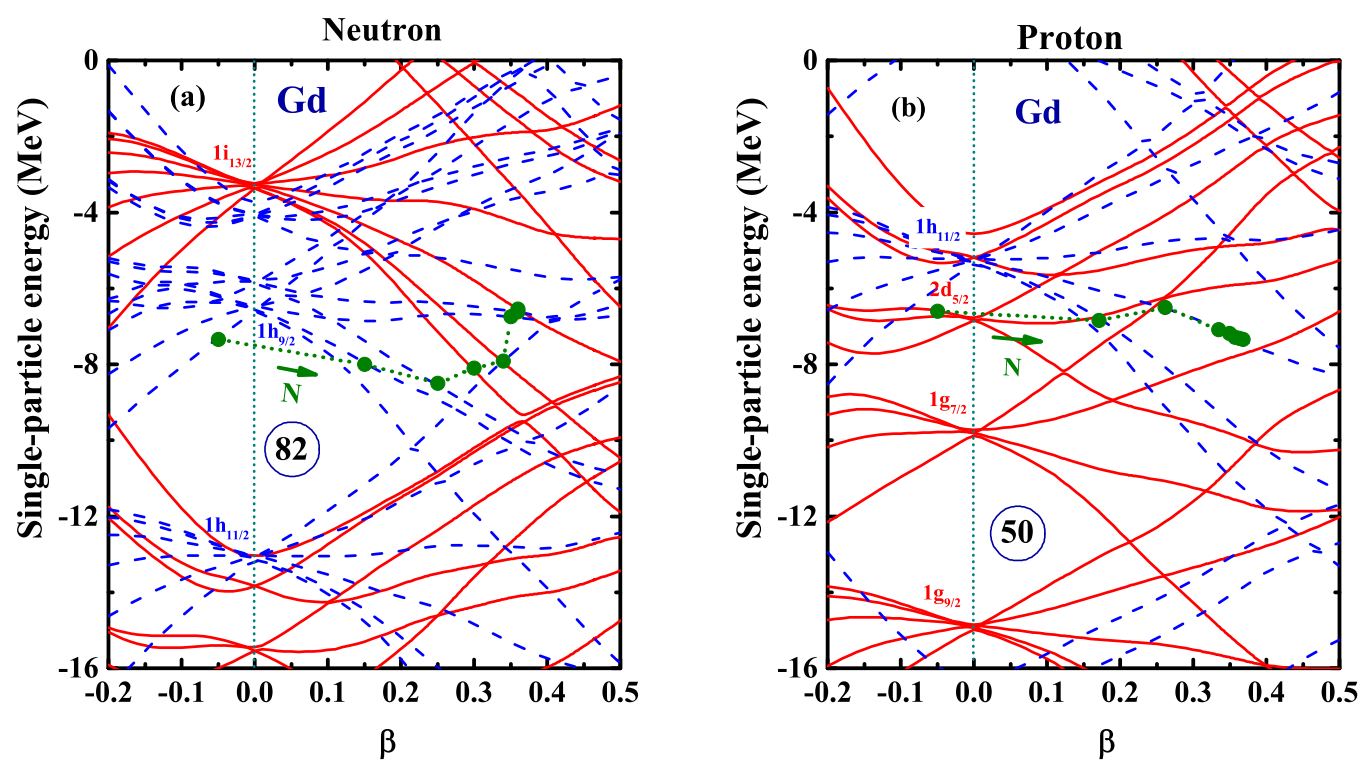

Fig. 10. The axially symmetric single neutron (left panel) and proton (right panel) levels of Gd as a function of the deformation parameter $\beta$. The red solid (blue dash) curves denote levels with positive (negative) parity. The green dots correspond to the positions of the last occupied levels at the minimum of the ground states in Gd isotopes.

2. D. Tonev, A. Dewald, T. Klug, P. Petkov, J. Jolie, A. Fitzler, O. Möller, S. Heinze, P. von Brentano, and R. F. Casten, Phys. Rev. C 69, 034334 (2004).

3. Z. Q. Sheng and J. Y. Guo, Mod. Phys. Lett. A 20, 2711 (2005).

4. R. Fossion, D. Bonatsos, and G. A. Lalazissis, Phys. Rev. C 73, 044310 (2006).

5. R. Rodríguez-Guzmán and P. Sarriguren, Phys. Rev. C 76, 064303 (2007).

6. L. M. Robledo, R. R. Rodríguez-Guzmán, and P. Sarriguren, Phys. Rev. C 78, 034314 (2008).

7. A. Leviatan, Phys. Rev. Lett. 77, 818 (1996).

8. P. Van Isacker, Phys. Rev. Lett. 83, 4269 (1999).

9. A. Leviatan and P. Van Isacker, Phys. Rev. Lett. 89, 222501 (2002).

10. A. Leviatan, Prog. Part. Nucl. Phys. 66, 93 (2011).

11. A. Leviatan, J. E. García-Ramos, and P. Van Isacker, Phys. Rev. C 87, 021302 (2013).

12. R. F. Casten, R. B. Cakirli, K. Blaum, and A. Couture, Phys. Rev. Lett. 113, 112501 (2014).

13. C. Bahri and D. Rowe, Nucl. Phys. A 662, 125 (2000).

14. D. Rowe, Nucl. Phys. A 745, 47 (2004).

15. C. Kremer, J. Beller, A. Leviatan, N. Pietralla, G. Rainovski, R. Trippel, and P. Van Isacker, Phys. Rev. C 89, 041302 (2014).

16. C. Kremer, J. Beller, A. Leviatan, N. Pietralla, G. Rainovski, R. Trippel, and P. Van Isacker, Phys. Rev. C 92, 039902 (2015).

17. P. Van Isacker, Nucl. Phys. News 24, 23 (2014).

18. P. Ring, Prog. Part. Nucl. Phys. 37, 193 (1996).

19. M. Bender, P. H. Heenen, and P. G. Reinhard, Rev. Mod. Phys. 75, 121 (2003).

20. D. Vretenar, A. Afanasjev, G. Lalazissis, and P. Ring, Phys. Rep. 409, 101 (2005).

21. J. Meng, H. Toki, S. G. Zhou, S. Q. Zhang, W. H. Long, and L. S. Geng, Prog. Part. Nucl. Phys. 57, 470 (2006).

22. J. Meng, Relativistic Density Functional for Nuclear Structure, vol. 10 (World Scientific, 2016).
23. T. Nikšić, Z. P. Li, D. Vretenar, L. Próchniak, J. Meng, and P. Ring, Phys. Rev. C 79, 034303 (2009).

24. T. Nikšić, D. Vretenar, and P. Ring, Prog. Part. Nucl. Phys. 66, 519 (2011).

25. Z. P. Li, T. Nikšić, D. Vretenar, J. Meng, G. Lalazissis, and P. Ring, Phys. Rev. C 79, 054301 (2009).

26. Z. P. Li, T. Nikšić, D. Vretenar, and J. Meng, Phys. Rev. C 80, 061301 (2009).

27. Z. P. Li, T. Nikšić, D. Vretenar, and J. Meng, Phys. Rev. C 81, 034316 (2010).

28. C. Y. Song, Z. P. Li, D. Vretenar, and J. Meng, Sci ChinaPhys Mech Astron 54, 222 (2011).

29. Z. P. Li, B. Y. Song, J. M. Yao, D. Vretenar, and J. Meng, Phys. Lett. B 726, 866 (2013).

30. Z. P. Li, J. M. Yao, D. Vretenar, T. Nikšić, H. Chen, and J. Meng, Phys. Rev. C 84, 054304 (2011).

31. J. Xiang, Z. P. Li, Z. X. Li, J. M. Yao, and J. Meng, Nucl. Phys. A 873, 1 (2012).

32. J. J. Sun, Z. Shi, X. Q. Li, H. Hua, C. Xu, Q. B. Chen, S. Q. Zhang, C. Y. Song, J. Meng, X. G. Wu, et al., Phys. Lett. B 734, 308 (2014).

33. T. Nikšić, P. Marević, and D. Vretenar, Phys. Rev. C 89, 044325 (2014).

34. Z. H. Wang, J. Xiang, W. H. Long, and Z. P. Li, J. Phys. G: Nucl. Part. Phys. 42, 045108 (2015).

35. Y. Y. Wang, Z. Shi, Q. B. Chen, S. Q. Zhang, C. Y. Song, Phys. Rev. C 93, 044309 (2016).

36. Z. P. Li, T. Nikšić, D. Vretenar, P. Ring, and J. Meng, Phys. Rev. C 81, 064321 (2010).

37. Z. P. Li, C. Y. Li, J. Xiang, J. M. Yao, and J. Meng, Phys. Lett. B 717, 470 (2012).

38. Y. Fu, H. Mei, J. Xiang, Z. Li, J. Yao, and J. Meng, Phys. Rev. C 87, 054305 (2013).

39. Z. Shi and S. Q. Zhang, A. Phys. Pol. B Supp. 8, 725 (2015).

40. P. W. Zhao, Z. P. Li, J. M. Yao, and J. Meng, Phys. Rev. C 82, 054319 (2010). 
41. L. Próchniak, K. Zajac, K. Pomorski, S. Rohoziński, and J. Srebrny, Nucl. Phys. A 648, 181 (1999).

42. http://www.nndc.bnl.gov/.

43. Z. P. Li, T. Nikšić, P. Ring, D. Vretenar, J. M. Yao, and J. Meng, Phys. Rev. C 86, 034334 (2012b).

44. Z. Podolyák, P. G. Bizzeti, A. M. Bizzeti-Sona, S. Lunardi, D. Bazzacco, A. Dewald, A. Algora, G. de Angelis, M. De Poli, E. Farnea, et al., Euro. Phys. J. A 8, 147 (2000).

45. Z. Podolyák, P. Bizzeti, A. Bizzeti-Sona, S. Lunardi, D. Bazzacco, A. Dewald, A. Algora, G. de Angelis, E. Farnea, A. Gadea, et al., Euro. Phys. J. A 17, 29 (2003).

46. N. V. Zamfir and R. F. Casten, Phys. Lett. B 260, 265 (1991).

47. E. A. McCutchan, D. Bonatsos, N. V. Zamfir, and R. F. Casten, Phys. Rev. C 76, 024306 (2007).

48. P. Garrett, M. Kadi, C. McGrath, V. Sorokin, M. Li, M. Yeh, and S. Yates, Phys. Lett. B 400, 250 (1997).

49. P. E. Garrett, J. Phys. G 27, R1 (2001).

50. L. Próchniak, P. Quentin, D. Samsoen, and J. Libert, Nucl. Phys. A 730, 59 (2004).

51. S. Piłat and K. Pomorski, Nucl. Phys. A 554, 413 (1993).

52. A. Couture, R. F. Casten, and R. B. Cakirli, Phys. Rev. C 91, 014312 (2015).

53. A. Bohr and B. R. Mottelson, Nuclear structure, vol. II (Benjamin, New York, 1975).

54. P. Ring and P. Schuck, The Nuclear Many-Body Problem (Springer-Verlag, 1980). 
